\title{
Algebraic Aspects of Spectral Theory
}

\author{
E B Davies
}

8 June 2010

\begin{abstract}
We describe some aspects of spectral theory that involve algebraic considerations but need no analysis. Some of the important applications of the results are to the algebra of $n \times n$ matrices with entries that are polynomials or more general analytic functions.
\end{abstract}

Short title: Algebraic Spectral Theory

MSC subject classification: 47A56, 47C05, 15A22, 16Sxx, 16Bxx

\section{Introduction}

This paper describes a range of results in spectral theory that can be formulated and proved in an algebraic context without assuming that the algebra has a norm or even that the underlying scalar field is the field of complex numbers. In some of the applications the 'scalars' involved are polynomials or rational functions. The author undertook this research in order to obtain some insights into a range of contexts in which such ideas are used, including some related to polynomial pencils, so-called non-linear spectral theory and control theory. Such problems are often not easily formulated in traditional Banach algebra terms. As well as its potential applications, indicated below, this study helps to delineate those aspects of spectral theory that do not depend on analytic tools - norms, continuity, complex analysis, etc.

A very limited account of spectral theory at our degree of generality may be found in [2, pp. 1-12, 87]. A fuller treatment is given in [4, Chap. 1], but it still has little overlap with the contents of this paper. More relevant is [23, Appendix 4], which gives an extensive account of various rings and fields of polynomials, analytic and meromorphic functions from the algebraic perspective. Some elements of this theory are described in the final section of the present paper. Simple examples of algebras to which the theorems in this paper might be applied are 
1. Algebras of bounded or unbounded operators on a Hilbert space and infinite tensor product algebras.

2. An important application is to the algebra $M(n, \mathcal{B})$ of all $n \times n$ matrices with entries in a commutative algebra $\mathcal{B}$, particularly the case in which $\mathcal{B}$ is the ring of polynomials (or rational functions) in a single complex variable. This example features in the theory of loop groups, [16, Sect. 3.5] and in the study of evolution equations that involve matrices with coefficients that depend polynomially or rationally on time.

3. The space $\mathcal{H}_{\infty}^{n}$ of all bounded, analytic, $n \times n$ matrix-valued functions on the upper half plane or the unit disc plays a key role in optimal control theory; see [13, 24]. References to the early literature on bounded operator-valued functions on the unit disc may be found in [20, Chap. 5]. In both cases one may regard the coefficient ring as the integral domain consisting of all bounded, complex-valued analytic functions on a domain in $\mathbf{C}$; the quotient field is a certain class of meromorphic functions on the same domain.

4. Voiculescu has developed an algebraic approach to non-commutative probability theory that has substantial applications to random matrix theory; see [22] and [21], where the term 'free probability theory' is used. In this application one considers $n \times n$ matrices whose entries lie in a certain algebra of unbounded random variables by using highly algebraic methods.

We emphasize that much of the material in this paper is classical if $\mathcal{A}$ is the algebra of all $n \times n$ complex matrices; see [12]. We are mainly concerned with the extent to which the results hold for more general choices of the algebra and base field. The final section describes some examples of relevant fields. We give a number of basic algebraic definitions in the paper for the benefit of those who are not familiar with the subject.

\section{Some elementary results}

Let $\mathcal{A}$ be an algebra over a field $\mathbf{F}$; we always assume that $\mathcal{A}$ contains an identity element, which we denote by $e$. We say that $\lambda \in \mathbf{F}$ does not lie in the spectrum of $a \in \mathcal{A}$ if there exists $b \in \mathcal{A}$ such that $(a-\lambda e) b=b(a-\lambda e)=e$. The spectrum of $a$ might be empty or equal to $\mathbf{F}$.

The formula $L_{a}(x)=a x$ defines a one-one algebra homomorphism from $\mathcal{A}$ to the algebra $\mathcal{L}(\mathcal{A})$ of linear operators on $\mathcal{A}$. The similar formula $R_{a}(x)=x a$ defines an anti-homomorphism, in the sense that $R_{a} R_{b}=R_{b a}$ for all $a, b \in \mathcal{A}$. 
The following lemma has some similarity to a corresponding result for $\mathrm{C}^{*}$ algebras, but it is applicable much more generally, for example to the algebra of $n \times n$ upper triangular matrices with entries in any field.

Lemma 1 We have $L_{a} R_{b}=R_{b} L_{a}$ for every $a, b \in \mathcal{A}$. If $M \in \mathcal{L}(\mathcal{A})$ and $M R_{b}=R_{b} M$ for all $b \in \mathcal{A}$ then there exists $a \in \mathcal{A}$ such that $M=L_{a}$.

Proof The first statement is elementary. To prove the second we put $a=M(e)$ and observe that

$$
M(b)=M\left(R_{b} e\right)=R_{b} M(e)=M(e) b=a b
$$

for all $b \in \mathcal{A}$, so $M=L_{a}$.

Lemma 2 If $L_{a}$ is invertible as an element of $\mathcal{L}(\mathcal{A})$ then a is an invertible element of $\mathcal{A}$ and $\left(L_{a}\right)^{-1}=L_{a^{-1}}$. Hence

$$
\operatorname{spec}\left(L_{a}\right)=\operatorname{spec}(a)
$$

for all $a \in \mathcal{A}$.

Proof Let $M \in \mathcal{L}(\mathcal{A})$ and

$$
M L_{a}(x)=x=L_{a} M(x)
$$

for all $x \in \mathcal{A}$. Putting $M(e)=b$ and $x=e$ in the second equality of (1) yields $e=a b$. Putting $x=b s$ in the first equality of (1) yields $M(s)=M(a b s)=$ $M L_{a}(b s)=b s$ for all $s \in \mathcal{A}$. Therefore $M=L_{b}$. Finally putting $x=e$ in the first equality of (11) yields $b a=L_{b} L_{a} e=e$ so $b=a^{-1}$.

We say that a non-zero element $a$ in an algebra $\mathcal{A}$ over $\mathbf{F}$ is algebraic if there exists a non-zero polynomial $p$ with coefficients in $\mathbf{F}$ such that $p(a)=0$. The set of all such polynomials is a non-zero, proper ideal $\mathcal{J}$ in the algebra $\mathcal{P}$ of all polynomials, and contains a unique monic polynomial $m$ of lowest degree, called the minimum polynomial of $a$. Moreover $\mathcal{J}=\{m q: q \in \mathcal{P}\}$. If $\mathcal{A}$ is finite-dimensional then all $a \in \mathcal{A}$ are algebraic, but there are important infinite-dimensional algebras in which the same holds.

Example 3 Let $\mathcal{A}_{r}=M\left(n_{r}, \mathbf{F}\right)$ for all positive integers $r$ and put $\mathcal{A}=$ $\bigotimes_{r=1}^{\infty} \mathcal{A}_{r}$. The algebra $\mathcal{A}$ is generated by expressions $x=\otimes_{r=1}^{\infty} x_{r}$ where all but a finite number of the $x_{r}$ equal the identity element of $\mathcal{A}_{r}$. Equivalently $\mathcal{A}=\bigcup_{m=1}^{\infty} \mathcal{B}_{m}$ where

$$
\mathcal{B}_{m}=\bigotimes_{r=1}^{m} \mathcal{A}_{r} \simeq M\left(n_{1} n_{2} \ldots n_{m}, \mathbf{F}\right) .
$$

Clearly every element of $\mathcal{A}$ is algebraic, but there is no upper bound on the degrees of the minimum polynomials. 
Theorem 4 Let a be an algebraic element of the subalgebra $\mathcal{A}$ of the algebra $\mathcal{B}$, where $\mathcal{A}$ and $\mathcal{B}$ have the same identity element e. If a has a left or right inverse $b$ in $\mathcal{B}$ then it has a two-sided inverse in $\mathcal{A}$. The spectrum of a in either $\mathcal{A}$ or to $\mathcal{B}$ equals the set of zeros of its minimum polynomial, and must be finite.

Proof Let $m$ be the minimum polynomial of $a$ and assume that $a$ has a right inverse $b \in \mathcal{B}$. If $m(\lambda)=\lambda^{n}+\sum_{r=0}^{n-1} \alpha_{r} \lambda^{r}$ and $\alpha_{0}=0$ then one would have

$$
\begin{aligned}
& a^{n-1}+\alpha_{n-1} a^{n-2}+\ldots+\alpha_{2} a+\alpha_{1} e \\
& \quad=\left(a^{n-1}+\alpha_{n-1} a^{n-2}+\ldots+\alpha_{2} a+\alpha_{1} e\right) a b \\
& =m(a) b \\
& \quad=0 .
\end{aligned}
$$

This contradicts the assumption that $m$ is the minimum polynomial, so we deduce that $\alpha_{0} \neq 0$. The same conclusion holds if we assume that $a$ has a left inverse in $\mathcal{B}$. It now follows immediately that

$$
a^{-1}=-\alpha_{0}^{-1}\left(a^{n-1}+\alpha_{n-1} a^{n-2}+\ldots+\alpha_{1} e\right) \in \mathcal{A} .
$$

The above calculations show that $a$ is invertible (in either $\mathcal{A}$ or $\mathcal{B}$ ) if and only if $m(0) \neq 0$. Since the minimum polynomial of $a-\lambda e$ is $m_{\lambda}(s)=m(s+\lambda)$, we conclude that $\lambda \in \operatorname{spec}(a)$ if and only if $m(\lambda)=0$.

It is sometimes useful to distinguish between the left and right spectra of an element $a$ of the algebra $\mathcal{A}$. One says that $\lambda \in \operatorname{spec}_{L}(a)$ if there does not exist $b \in \mathcal{A}$ such that $b(\lambda e-a)=e$, and that $\lambda \in \operatorname{spec}_{R}(a)$ if there does not exist $c \in \mathcal{A}$ such that $(\lambda e-a) c=e$. One sees immediately that

$$
\operatorname{spec}(a)=\operatorname{spec}_{L}(a) \cup \operatorname{spec}_{R}(a) .
$$

The elements $b, c$ above need not be unique. Indeed, if there is a unique solution $b \in \mathcal{A}$ of $a b=e$ then the identity

$$
a(b+b a-e)=a b+(a b) a-a=e
$$

implies that $b$ is the two-sided inverse of $a$.

Lemma 5 If $a$ is an algebraic element of $\mathcal{A}$ then

$$
\operatorname{spec}_{L}(a)=\operatorname{spec}_{R}(a)=\operatorname{spec}(a)
$$

for all $a \in \mathcal{A}$. 
Proof Let $m$ be the minimum polynomial of $\lambda e-a$. If $\lambda \notin \operatorname{spec}_{L}(a)$ then there exists $b \in \mathcal{A}$ such that $b(\lambda e-a)=e$. Since $\lambda e-a$ is algebraic, Theorem 4 implies that $\lambda \notin \operatorname{spec}(a)$. Hence $\operatorname{spec}(a) \subseteq \operatorname{spec}_{L}(a)$. The inclusion $\operatorname{spec}(a) \subseteq \operatorname{spec}_{R}(a)$ has a similar proof and the reverse inclusions are elementary.

Example 6 The condition in Lemma 5 that the element $a$ is algebraic cannot be omitted. Let $\mathcal{A}$ be the algebra of all bounded linear operators on the Hilbert space $\ell^{2}\left(\mathbf{Z}^{+}\right)$and let $a \in \mathcal{A}$ be defined by $(a \phi)(n)=\phi(n+1)$ for all $\phi \in \ell^{2}\left(\mathbf{Z}^{+}\right)$ and all $n \geq 1$. Then

$$
\begin{aligned}
\operatorname{spec}_{L}(a) & =\{z \in \mathbf{C}:|z| \leq 1\} \\
\operatorname{spec}_{R}(a) & =\{z \in \mathbf{C}:|z|=1\} .
\end{aligned}
$$

The proof of the first identity uses $(\lambda e-a) \phi_{\lambda}=0$ whenever $|\lambda|<1$, where $\phi_{\lambda} \in$ $\ell^{2}\left(\mathbf{Z}^{+}\right)$is defined by $\phi_{\lambda}(n)=\lambda^{n}$ for all $n \geq 1$. The proof of the second identity uses $a a^{*}=e$ and $(\lambda e-a) c_{\lambda}=e$ whenever $|\lambda|<1$, where $c_{\lambda}=-a^{*}\left(e-\lambda a^{*}\right)^{-1}$.

We next prove a spectral mapping theorem for polynomials.

Theorem 7 If the field $\mathbf{F}$ is algebraically closed, $a \in \mathcal{A}$ and $p$ is a polynomial then

$$
\operatorname{spec}(p(a))=\{p(z): z \in \operatorname{spec}(a)\} .
$$

Proof Suppose that $p$ is monic and of degree $n$. The statement of the theorem can be rewritten in several ways each of which is equivalent to the next one.

- Given $y \in \mathbf{F}$ then $y \in \operatorname{spec}(p(a))$ if and only if there exists $x \in \operatorname{spec}(a)$ such that $y=p(x)$.

- Let

$$
q(s)=p(s)-y=\prod_{r=1}^{n}\left(s-s_{r}\right),
$$

where $s_{r}$ depend on $y$. Then $0 \in \operatorname{spec}(q(a))$ if and only if there exists $x \in \operatorname{spec}(a)$ such that $q(x)=0$.

- $0 \in \operatorname{spec}(q(a))$ if and only if there exists $x \in \operatorname{spec}(a)$ and $r \in\{1, \ldots, n\}$ such that $x-s_{r}=0$.

- $0 \in \operatorname{spec}(q(a))$ if and only if there exists $r \in\{1, \ldots, n\}$ such that $s_{r} \in$ $\operatorname{spec}(a)$.

- $q(a)$ is not invertible if and only if there exists $r \in\{1, \ldots, n\}$ such that $a-s_{r} e$ is not invertible. 
- $q(a)$ is invertible if and only if $a-s_{r} e$ is invertible for all $r \in\{1, \ldots, n\}$.

The truth of the final statement is an elementary consequence of the fact that $q(a)$ is the product of the $a-s_{r} e$, all of which commute in $\mathcal{A}$.

Without the hypothesis that $\mathbf{F}$ is algebraically closed, the theorem is false. For example if $\mathbf{F}=\mathbf{R}$ and $a=\left(\begin{array}{cc}1 & 1 \\ -1 & 1\end{array}\right)$ then $\operatorname{spec}(a)=\emptyset \operatorname{but} \operatorname{spec}\left(a^{4}\right)=\{-4\}$.

\section{Commutative Algebras}

In this section we present an algebraic version of Gelfand's representation of commutative Banach algebras as function algebras.

Let $\mathcal{A}$ be a commutative algebra with identity $e$ over a field $\mathbf{F}$ and let $J_{m}$ denote its maximal proper ideals, which parametrized by $m \in M$. For each $m \in M, \mathbf{F}_{m}=\mathcal{A} / J_{m}$ is a commutative algebra with no proper ideals and hence is a field containing $\mathbf{F}$. The natural homomorphism $\phi_{m}: \mathcal{A} \rightarrow \mathbf{F}_{m}$ is called a character of $\mathcal{A}$.

The set $M$ of all maximal ideals in $\mathcal{A}$ is called the max-spectrum of $\mathcal{A}$ in algebraic geometry to distinguish it from the spectrum, namely the set $N$ of all prime ideals. Elements of $M$ correspond to algebra homomorphisms mapping $\mathcal{A}$ onto a field, which we call characters, while elements of $N$ correspond to algebra homomorphisms mapping $\mathcal{A}$ into a field, or equivalently onto an integral domain. Every maximal ideal is obviously prime.

Determining the maximal ideals, prime ideals or characters in an algebra is a highly non-trivial matter. Hilbert's Nullstellensatz implies that if $\mathcal{A}$ is the algebra of polynomials on $\mathbf{C}^{n}$ then every character is obtained by evaluation at some point of $\mathbf{C}^{n}$; see [1, p. 85]. For some other algebras of unbounded continuous functions this is not true and the question is much harder.

The Gelfand formula

$$
\widehat{a}(m)=\phi_{m}(a)
$$

takes an element $a \in \mathcal{A}$ and maps it by an algebra homomorphism to $\widehat{a} \in$ $\mathcal{F}(M)$, where $\mathcal{F}(M)$ is the algebra of all functions $f$ on $M$ such that $f(m) \in$ $\mathbf{F}_{m}$. (More correctly $\mathcal{F}(M)$ is the algebra of sections of a certain bundle over M.)

Theorem 8 The element $a \in \mathcal{A}$ is invertible if and only if $\widehat{a}(m) \neq 0$ for all $m \in M$. If $a \in \mathcal{A}$ then

$$
\operatorname{spec}(a)=\mathbf{F} \cap\{\widehat{a}(m): m \in M\} .
$$


Proof If $a$ is invertible and $a b=e$ then

$$
\widehat{a}(m) \widehat{b}(m)=\phi_{m}(a) \phi_{m}(b)=\phi_{m}(a b)=e,
$$

so $\widehat{a}(m) \neq 0$ for every $m \in M$. Conversely if $a$ is not invertible then $a \mathcal{A}$ is a proper ideal in $\mathcal{A}$ and is contained in some maximal proper ideal $J_{m}$. Since $a \in J_{m}$ one has $\widehat{a}(m)=0$.

The final statement of the theorem is proved by applying the above to $a-\lambda e$ for every choice of $\lambda \in \mathbf{F}$.

Example 9 Let $I=[0,1]$ and let $\mathcal{A}_{1}$ be the algebra over $\mathbf{Q}$ consisting of all continuous functions $f: I \rightarrow \mathbf{R}$ such that $f(\mathbf{Q} \cap I) \subseteq \mathbf{Q}$, where $\mathbf{Q}$ is the field of rational numbers. Given a positive integer $n$ and any sequence $a_{0}, a_{1}, \ldots, a_{n} \in \mathbf{Q}$ one may define a function $f \in \mathcal{A}_{1}$ by linear interpolation starting from the values $f(r / n)=a_{r}$ where $0 \leq r \leq n$. This construction may be used to prove that $\mathcal{A}_{1}$ is uniformly dense in the algebra of all continuous real-valued functions on $I$.

For each $a \in I$ one may define the character $\phi_{a}$ on $\mathcal{A}_{1}$ by

$$
\phi_{a}(f)=f(a) .
$$

One notes that $\mathbf{F}_{a}=\mathbf{Q}$ if $a \in \mathbf{Q} \cap I$ but $\mathbf{F}_{a}=\mathbf{R}$ otherwise.

Theorem 10 Every character $\phi$ of $\mathcal{A}_{1}$ is of the form $\phi_{a}$ for some $a \in I$.

Proof Suppose that $\phi$ is not of the stated form. For every $a \in I$ the kernels $J$ and $J_{a}$ of $\phi$ and $\phi_{a}$ respectively are maximal proper ideals, so there exists $f_{a} \in J$ such that $f_{a}(a) \neq 0$. By a compactness argument there exist $a_{1}, \ldots, a_{m} \in I$ such that $h:=\sum_{r=1}^{m}\left|f_{a_{r}}\right|^{2}>0$ on $I$. This yields a contradiction, because $h \in J$ and a proper ideal cannot contain an invertible element.

Example 11 The algebra $\mathcal{A}_{2}=C_{b}(\mathbf{R})$ of all bounded, continuous, complexvalued functions $f$ on $\mathbf{R}$ is a $\mathrm{C}^{*}$-algebra for the norm

$$
\|f\|=\sup \{|f(x)|: x \in \mathbf{R}\},
$$

and for no other norm; see [4, Theorem 3.2.6]. The characters of $\mathcal{A}_{2}$ are all norm continuous and are in one-one correspondence with the points in the Stone-Cech compactification of $\mathbf{R}$ and all characters are complex-valued; see [4, Def. 4.2.6]. The only characters that can be identified constructively are those given by the formula (2) where $a \in \mathbf{R}$.

Example 12 The algebra $\mathcal{A}_{3}=C(\mathbf{R})$ of all continuous, complex-valued functions on $\mathbf{R}$ has 'finite' characters given by (2). We say that a character $\phi$ on $\mathcal{A}_{3}$ is 'at infinity' if it is not of this form. Lemma 13 and Theorem 14] give some insight into the form of the characters at infinity. 
Lemma 13 Every character $\phi$ at infinity on $\mathcal{A}_{3}$ has a kernel $J_{\phi}$ that contains the ideal

$$
J=\left\{f \in \mathcal{A}_{3}: \operatorname{supp}(f) \text { is compact }\right\} .
$$

Proof Suppose that $f \in \mathcal{A}_{3}$ has support contained in $(-n, n)$ for some $n$. Let $a \in[-n, n]$. Since $J_{\phi}$ and $J_{a}=\left\{f \in \mathcal{A}_{3}: f(a)=0\right\}$ are both maximal proper ideals, there exists $f_{a} \in J_{\phi}$ such that $f_{a}(a) \neq 0$. By a compactness argument there exist $a_{1}, \ldots, a_{m} \in[-n, n]$ such that $h:=\sum_{r=1}^{m}\left|f_{a_{r}}\right|^{2}>0$ on $[-n, n]$. Since $f=h k$ for some $k \in \mathcal{A}_{3}$, it follows that $f \in J_{\phi}$. This proves the lemma.

Theorem 14 If $\phi$ is a character of $\mathcal{A}_{3}$ at infinity then the field $\phi(\mathcal{A})$ contains the field $G$ of all complex-valued rational functions.

Proof Every $g \in G$ has a finite number of poles, so there exists a smallest integer $n \geq 0$ such that $g(x)$ is continuous on $\{x \in \mathbf{R}:|x| \geq n\}$. We define $\rho(g) \in \mathcal{A}_{3}$ to be equal to $g$ on $\{x \in \mathbf{R}:|x| \geq n\}$ and by linear interpolation in $[-n, n]$.

If $J$ is defined by (3), then

$$
\rho\left(g_{1}\right)+\rho\left(g_{2}\right)-\rho\left(g_{1}+g_{2}\right) \in J, \quad \rho\left(g_{1}\right) \rho\left(g_{2}\right)-\rho\left(g_{1} g_{2}\right) \in J
$$

for all $g_{1}, g_{2} \in G$. Therefore $g \rightarrow \rho(g)+J$ defines a one-one structure preserving map from $G$ into $\mathcal{A} / J$. By an abuse of notation one may say that $G \subseteq \mathcal{A} / J$.

There is a one-one correspondence between ideals in $\mathcal{A} / J$ and ideals in $\mathcal{A}$ that contain $J$. Similarly, the characters on $\mathcal{A}$ whose kernels contain $J$ may be identified with the characters on the algebra $\mathcal{A} / J$. The restriction of such characters to $G$, regarded as a subfield of $\mathcal{A} / J$, must be one-one, because a field contains no proper ideals, so the field $\phi(\mathcal{A} / J)$ must contain a copy of $G$.

\section{Resolvent families}

We now turn to the study of (pseudo) resolvent families; the ideas here are taken from [6]. From this point onwards $\mathcal{A}$ will denote a (typically non-commutative) algebra over a field $\mathbf{F}$. A resolvent family $(r, S)$ in $\mathcal{A}$ consists of a set $S \subseteq \mathbf{F}$ together with a map $r: S \rightarrow \mathcal{A}$ such that

$$
r_{\lambda}-r_{\mu}=(\mu-\lambda) r_{\lambda} r_{\mu}
$$

for all $\lambda, \mu \in S$. By interchanging the role of $\lambda, \mu$ one readily sees that this implies that $r_{\lambda}$ and $r_{\mu}$ commute for all $\lambda, \mu \in S$. We say that $(\widetilde{r}, \widetilde{S})$ extends 
$(r, S)$ if $S \subseteq \widetilde{S}$ and $\widetilde{r}(\lambda)=r(\lambda)$ for all $\lambda \in S$. We say that $(r, S)$ is maximal if it has no proper extension.

If $a \in \mathcal{A}$ and $S=\mathbf{F} \backslash \operatorname{spec}(a)$ then one obtains a (true) resolvent family by putting $r_{\lambda}=(\lambda e-a)^{-1}$ for all $\lambda \in S$. (Pseudo) resolvent families are not all of this type; if $a$ is an unbounded closed linear operator on a Banach space $\mathcal{B}$ and $\mathcal{A}$ is the algebra of all bounded operators on $\mathcal{B}$ then one may define the resolvent family associated with $a$ just as in the case in which $a$ is bounded. The theory of such resolvent families is very highly developed because of the role it plays in the theory of differential operators and one-parameter semigroups, [5].

Theorem 15 A resolvent family $(r, S)$ is the family associated with an element $a \in \mathcal{A}$ if and only if it is maximal and $r_{\lambda}$ is invertible for some (equivalently all) $\lambda \in S$. If this holds then $\operatorname{spec}(a)=\mathbf{F} \backslash S$.

Proof Suppose that $b r_{\mu}=r_{\mu} b=e$ for some $b \in \mathcal{A}$ and $\mu \in S$. Multiplying (4) on the left (right) by $b$ yields $e=(b-\mu e+\lambda e) r_{\lambda}=r_{\lambda}(b-\mu e+\lambda e)$, so $r_{\lambda}$ is invertible for all $\lambda \in S$. Putting $a=\mu e-b$ then yields $r_{\lambda}=(\lambda e-a)^{-1}$ for all $\lambda \in S$.

Given $a \in \mathcal{A}$ let $\left(r_{0}, S_{0}\right)$ be the resolvent family associated with $a$, where $S_{0}=$ $\mathbf{F} \backslash \operatorname{spec}(a)$. Suppose that it is not maximal, that $(r, S)$ is a proper extension and that $\lambda \in S \cap \operatorname{spec}(a)$. Then $r_{\lambda}$ is invertible with inverse which we denote $b$ and $r_{\mu}=(\mu e-a)^{-1}$ for some chosen $\mu \in S$. If we multiply (4) on the left by $b$ and on the right by $\mu e-a$ we obtain

$$
\mu e-a-b=(\mu-\lambda) e .
$$

Therefore $b=\lambda e-a$; since $b$ is invertible we conclude that $\lambda \notin \operatorname{spec}(a)$. The contradiction implies that $\left(r_{0}, S_{0}\right)$ is maximal.

Theorem 16 Every resolvent family $(r, S)$ is uniquely determined on $S$ by $r_{\mu}$ for a single chosen $\mu \in S$. Moreover $(r, S)$ has a unique maximal extension $(\widetilde{r}, \widetilde{S})$.

Proof Given $\alpha, \lambda \in S$ the resolvent equation implies that

$$
\left(e+(\lambda-\alpha) r_{\alpha}\right)\left(e+(\alpha-\lambda) r_{\lambda}\right)=e .
$$

Therefore $e+(\lambda-\alpha) r_{\alpha}$ is invertible and

$$
r_{\lambda}=(\alpha-\lambda)^{-1}\left\{\left(e+(\lambda-\alpha) r_{\alpha}\right)^{-1}-e\right\}=\left(e+(\lambda-\alpha) r_{\alpha}\right)^{-1} r_{\alpha} .
$$

If we define

$$
\widetilde{r}_{\lambda}=\left(e+(\lambda-\alpha) r_{\alpha}\right)^{-1} r_{\alpha}
$$


on the set $\widetilde{S}$ of all $\lambda \in \mathbf{F}$ for which $e+(\lambda-\alpha) r_{\alpha}$ is invertible, then $\widetilde{r}$ must be the maximal extension of $r$ provided $\widetilde{r}$ satisfies the resolvent equation. If $\lambda, \mu \in \widetilde{S}$ then

$$
\begin{aligned}
(\mu-\lambda) \widetilde{r}_{\lambda} \widetilde{r}_{\mu}= & (\mu-\lambda) r_{\alpha}\left(e+(\lambda-\alpha) r_{\alpha}\right)^{-1}\left(e+(\lambda-\alpha) r_{\alpha}\right)^{-1} r_{\alpha} \\
= & \left\{\left(e+(\mu-\alpha) r_{\alpha}\right)-\left(e+(\lambda-\alpha) r_{\alpha}\right)\right\} \\
& .\left(e+(\lambda-\alpha) r_{\alpha}\right)^{-1}\left(e+(\mu-\alpha) r_{\alpha}\right)^{-1} r_{\alpha} \\
= & \left\{\left(e+(\lambda-\alpha) r_{\alpha}\right)^{-1}-\left(e+(\mu-\alpha) r_{\alpha}\right)^{-1}\right\} r_{\alpha} \\
= & \widetilde{r}_{\lambda}-\widetilde{r}_{\mu} .
\end{aligned}
$$

Motivated by the last two theorems, we define the $\operatorname{spectrum} \operatorname{spec}(r, S)$ of a resolvent family $(r, S)$ to be $\mathbf{F} \backslash \widetilde{S}$ where $(\widetilde{r}, \widetilde{S})$ is its unique maximal extension. Our next task is to explain how one can use algebraic ideas to classifying the spectrum into parts.

If $\mathcal{J}$ is a two-sided ideal in $\mathcal{A}$, there is a natural algebra homomorphism $\pi_{\mathcal{J}}$ from $\mathcal{A}$ onto the quotient algebra $\mathcal{A} / \mathcal{J}$. Given $a \in \mathcal{A}$ we put

$$
\begin{aligned}
\operatorname{spec}_{\mathcal{J}}(a) & =\operatorname{spec}\left(\pi_{\mathcal{J}}(a)\right) \\
\operatorname{spec}_{L, \mathcal{J}}(a) & =\operatorname{spec}_{L}\left(\pi_{\mathcal{J}}(a)\right) \\
\operatorname{spec}_{R, \mathcal{J}}(a) & =\operatorname{spec}_{R}\left(\pi_{\mathcal{J}}(a)\right) .
\end{aligned}
$$

See [8, Chap. 1.3] for a full discussion of the various definitions of the essential spectrum of an operator on a Hilbert space $\mathcal{H}$; one widely used definition arises by taking $\mathcal{A}$ to be the algebra of all bounded operators on $\mathcal{H}$ and $\mathcal{J}$ to be the ideal of all compact operators. The quotient algebra $\mathcal{A} / \mathcal{J}$ is called the Calkin algebra and arises in the study of Fredholm operators and index theory.

Lemma 17 Let $(r, S)$ be a maximal resolvent family in $\mathcal{A}$. If one defines

$$
\operatorname{spec}_{\mathcal{J}}(r, S)=\operatorname{spec}\left(\pi_{\mathcal{J}} r, S\right)
$$

then

$$
\operatorname{spec}_{\mathcal{J}}(r, S) \subseteq \operatorname{spec}(r, S)
$$

Proof One sees immediately that $\left(\pi_{\mathcal{J}} r, S\right)$ is a resolvent family in $\mathcal{A} / \mathcal{J}$. If $\widetilde{S}$ is the domain of its maximal extension then the lemma is simply a restatement of the fact that $S \subseteq \widetilde{S}$.

Note that if $(r, S)$ is associated with a closed unbounded operator $a$ on $\mathcal{H}$, then the resolvent family $\left(\pi_{\mathcal{J}} r, S\right)$ has a much less direct relationship with $a$ because $\pi_{\mathcal{J}} a$ is not easy to define. 
We turn to some applications to perturbation theory. The algebra $\mathcal{L}(\mathcal{H})$ of all bounded operators on a Hilbert space $\mathcal{H}$ contains many two-sided ideals, but the set $\mathcal{C}_{\infty}$ of all compact operators and the set $\mathcal{C}_{1}$ of all trace class operators are particularly important. The following results are abstractions of theorems known in the above context; they have proved important in quantum theory, because of the existence of physically relevant $C^{*}$-algebras with many twosided ideals. See [6, 10, 11] for many references to these recent developments. These ideals allow the division of the classical essential spectrum into parts with different natures.

Let $\mathcal{J}$ be a two-sided ideal in an algebra $\mathcal{A}$ over the field $\mathbf{F}$. If $a, b \in \mathcal{A}$ and $\lambda \notin \operatorname{spec}(a) \cup \operatorname{spec}(b)$ then the identity

$$
(\lambda e-a)^{-1}-(\lambda e-b)^{-1}=(\lambda e-a)^{-1}(a-b)(\lambda e-b)^{-1}
$$

implies that $a-b \in \mathcal{J}$ if and only if the difference of the two resolvents lies in $\mathcal{J}$. Moreover the definition of the $\mathcal{J}$-spectrum implies immediately that $\operatorname{spec}_{\mathcal{J}}(a)=\operatorname{spec}_{\mathcal{J}}(b)$ provided $a-b \in \mathcal{J}$.

If $A, B$ are two unbounded closed operators on a Hilbert space $\mathcal{H}$ the analogous result is proved by treating the resolvent operators directly as follows; see [8, Theorem IX.2.4], where $\mathcal{J}$ is the ideal of compact operators in $\mathcal{L}(\mathcal{H})$.

Theorem 18 Let $\left(r_{1}, S_{1}\right)$ and $\left(r_{2}, S_{2}\right)$ be two resolvent families in $\mathcal{A}$ and let $S=S_{1} \cap S_{2}$. If $r_{1, \lambda}-r_{2, \lambda}$ lies in the two-sided ideal $\mathcal{J}$ for some $\lambda \in S$ then this holds for all $\lambda \in S$ and $\operatorname{spec}_{\mathcal{J}}\left(r_{1}\right)=\operatorname{spec}_{\mathcal{J}}\left(r_{2}\right)$.

Proof If we put $\widetilde{r}_{i}=\pi_{\mathcal{J}}\left(r_{i}\right)$ then the hypothesis implies that $\widetilde{r}_{1, \lambda}=\widetilde{r}_{2, \lambda}$ for some $\lambda \in S$. Theorem 16 now implies that $\widetilde{r}_{1}=\widetilde{r}_{2}$ throughout $S$. Therefore they have the same maximal extension and the original resolvents have the same $\mathcal{J}$-spectrum.

The following theorem is adapted from [15, Lemma 10.1.8], where applications to the essential spectra of Hankel operators are given.

Theorem 19 If $a_{1}, \ldots, a_{n} \in \mathcal{A}$ and $a_{r} a_{s} \in \mathcal{J}$ for all $r \neq s$ then

$$
\operatorname{spec}_{\mathcal{J}}(a) \cup\{0\}=\bigcup_{r=1}^{n} \operatorname{spec}_{\mathcal{J}}\left(a_{r}\right)
$$

where $a=\sum_{r=1}^{n} a_{r}$.

Proof We put $\widetilde{x}=\pi_{\mathcal{J}} x \in \mathcal{A} / \mathcal{J}$ for every $x \in \mathcal{A}$. The hypotheses imply that $\widetilde{a}_{r} \widetilde{a}_{s}=0$ for all $r \neq s$ and hence that

$$
\left(\lambda \widetilde{e}-\widetilde{a}_{1}\right) \ldots\left(\lambda \widetilde{e}-\widetilde{a}_{n}\right)=\lambda^{n-1}(\lambda \widetilde{e}-\widetilde{a})
$$


for all $\lambda \in \mathbf{F}$. Since all the terms in this equation commute we conclude that if $\lambda \neq 0$ then $\lambda \widetilde{e}-\widetilde{a}$ is invertible if and only if $\lambda \widetilde{e}-\widetilde{a}_{r}$ are invertible for all $r$. This completes the proof as far as non-zero $\lambda$ are concerned.

If $0 \notin \operatorname{spec}\left(\widetilde{a}_{1}\right)$ then $\widetilde{a}_{1}$ is invertible and the identity $\widetilde{a}_{1} \widetilde{a}_{r}=0$ implies that $\widetilde{a}_{r}=0$ for all $r \neq 1$. Therefore $0 \in \bigcup_{r=1}^{n} \operatorname{spec}\left(\widetilde{a}_{r}\right)$ in all cases.

Example 20 The following application of Theorem 19 is well-known in the spectral analysis of Schrödinger operators. Let $p_{1}, \ldots, p_{n} \in \mathcal{A}$ satisfy $p_{r} p_{s}=$ $\delta_{r, s} p_{r}$ for all $r, s$ and $\sum_{r=1}^{n} p_{r}=e$. Let $a \in \mathcal{A}$ and assume that $a_{r, s}:=p_{r} a p_{s} \in \mathcal{J}$ for all $r \neq s$. Then by applying Theorem 19 to the double sum $a=\sum_{r, s} a_{r, s}$ one obtains

$$
\operatorname{spec}_{\mathcal{J}}(a) \cup\{0\}=\bigcup_{r=1}^{n} \operatorname{spec}_{\mathcal{J}}\left(a_{r, r}\right) .
$$

Example 21 Let $\mathcal{A}$ be the algebra $M(n, \mathcal{P})$ of all $n \times n$ matrices whose entries are polynomials in a variable $x \in \mathbf{F}$. The spectrum of an element of $\mathcal{A}$ is normally equal to $\mathbf{F}$ because an element of $\mathcal{A}$ is invertible if and only if its determinant is a non-zero constant. However, if $n=2$ and

$$
a=\left(\begin{array}{cc}
1 & 1 \\
x & 1+x
\end{array}\right), \quad b=\left(\begin{array}{cc}
0 & 1+x^{3} \\
0 & 0
\end{array}\right)
$$

$x \in \mathbf{F}$ being the polynomial variable, then $\operatorname{spec}(a)=\mathbf{F} \backslash\{0\}$ while $\operatorname{spec}(b)=$ $\{0\}$.

By passing to suitable quotient algebras one obtains more interesting spectra. Let $\mathcal{A}=M(n, \mathcal{P})$ and let $S$ be a finite subset of $\mathbf{F}$. We define $\mathcal{B}_{S}$ to be the algebra of all functions $f: S \rightarrow M(n, \mathbf{F})$ with the obvious pointwise operations and define $\pi_{S}: \mathcal{A} \rightarrow \mathcal{B}_{S}$ by $\pi_{S}(a)=f$ where $f(s)=a(s)$ for all $s \in S$. The kernel of $\pi_{S}$ is a two-sided ideal $\mathcal{J}_{S}$ in $\mathcal{A}$ and $\pi_{S}$ is an algebra homomorphism from $\mathcal{A}$ onto $\mathcal{B}_{S}$. Moreover

$$
\operatorname{spec}_{\mathcal{J}_{S}}(a)=\operatorname{spec}\left(\pi_{S} a\right)=\bigcup_{s \in S} \operatorname{spec}(a(s)) .
$$

for all $a \in \mathcal{A}$. Hence $\#\left(\operatorname{spec}_{\mathcal{J}_{S}}(a)\right) \leq n(\# S)$. As $S$ increases, the kernels of the ideals $\mathcal{J}_{S}$ decrease and $\operatorname{spec}_{\mathcal{J}_{S}}(a)$ increases to $\bigcup_{x \in \mathbf{F}} \operatorname{spec}(a(x))$.

If one puts $\mathbf{F}=\mathbf{R}, n=2$ and defines $a \in \mathcal{A}$ by

$$
a=\left(\begin{array}{cc}
x^{2} & 1 \\
0 & x^{2}
\end{array}\right)
$$

then $\operatorname{spec}(a)=\mathbf{R}$ but $\bigcup_{x \in \mathbf{R}} \operatorname{spec}(a(x))=[0, \infty)$. 


\section{Connections between $a b$ and $b a$}

If $a, b$ lie in an algebra $\mathcal{A}$ over $\mathbf{F}$, an elementary calculation shows that

$$
b p(a b)=p(b a) b
$$

for every polynomial $p$ with coefficients in $\mathbf{F}$. The calculations on this section are based on the idea that this relationship might be valid for other classes of function. The following theorem may be found in [4, Prop. 1.5.29].

Lemma 22 If $a, b \in \mathcal{A}$ then

$$
\operatorname{spec}(a b) \cup\{0\}=\operatorname{spec}(b a) \cup\{0\} .
$$

If $\operatorname{dim}(\mathcal{A})<\infty$ then

$$
\operatorname{spec}(a b)=\operatorname{spec}(b a) .
$$

Moreover $a b$ is invertible if and only if both $a$ and $b$ are invertible.

Proof If $\lambda \neq 0$ and $\lambda e-a b$ is invertible then a direct calculation shows that

$$
c(\lambda e-b a)=(\lambda e-b a) c=e
$$

where

$$
c=\lambda^{-1}\left(e+b(\lambda e-a b)^{-1} a\right) .
$$

The first part of the lemma follows by interchanging the roles of $a$ and $b$.

In order to prove the second part we have to show that $a b$ is invertible if and only if $b a$ is invertible, whenever $\operatorname{dim}(\mathcal{A})<\infty$. If $a b$ is invertible then there exists $c \in \mathcal{A}$ such that $(a b) c=e$. Therefore $L_{a} L_{b} L_{c}=I$ so

$$
\operatorname{det}\left(L_{a}\right) \operatorname{det}\left(L_{b}\right) \operatorname{det}\left(L_{c}\right)=1 .
$$

It follows that $L_{a}, L_{b}$ are invertible. Lemma 2 now implies that $a, b$ are invertible and hence that $b a$ is invertible. The converse is similar.

Example 23 The following arises in the theory of quantum groups, more specifically the quantum torus. If $a, b \in \mathcal{A}$ and $a b=z b a$ for some non-zero $z \in \mathbf{F}$ then Lemma 22 implies that $\operatorname{spec}(a b)$ is invariant under multiplication by $z^{ \pm 1}$. If $\mathcal{A}$ is a Banach algebra then the boundedness of the spectrum of $a b$ further implies that $|z|=1$.

We say that $J \subseteq \mathcal{A}$ is a right ideal if it is a linear subspace of $\mathcal{A}$ and $x y \in J$ whenever $x \in J$ and $y \in \mathcal{A}$. If $a \in \mathcal{A}$ one defines the principal right ideal $J_{a}$ 
by $J_{a}=\{a x: x \in \mathcal{A}\}$ then $J_{a}=J_{b}$ if and only if there exist $x, y \in \mathcal{A}$ such that $a x=b$ and $b y=a$.

If $\mathcal{A}$ is an algebra of linear maps on $V$ and $W$ is a linear subspace of $V$, then one may define a right ideal of $\mathcal{A}$ by

$$
J_{W}=\{x: x(V) \subseteq W\} .
$$

If $\lambda \in \mathbf{F}$ lies in the spectrum of $a \in \mathcal{A}$ then one may define a class of 'Jordan' right ideals $J(a, \lambda, n)$ by

$$
J(a, \lambda, n)=\left\{x \in \mathcal{A}:(a-\lambda e)^{n} x=0\right\} .
$$

Lemma 24 Under the above assumptions

$$
J(a, \lambda, n) \subseteq J(a, \lambda, n+1)
$$

for all natural numbers $n$. Either all $J(a, \lambda, n)$ are distinct or there exists $N$ such that $J(a, \lambda, n) \neq J(a, \lambda, n+1)$ if $n<N$ but $J(a, \lambda, n)=J(a, \lambda, n+1)$ if $n \geq N$. If $\operatorname{dim}(\mathcal{A})<\infty$ then the latter case occurs.

Lemma 25 If $a, b \in \mathcal{A}$ and $\lambda \neq 0$ then there is a natural isomorphism of $J(a b, \lambda, n)$ with $J(b a, \lambda, n)$ for all $n$.

Proof We start by putting $J_{1}=J(b a, \lambda, n)$ and $J_{2}=J(a b, \lambda, n)$. If $x \in J_{1}$ then

$$
(a b-\lambda e)^{n} a x=a(b a-\lambda e)^{n} x=0
$$

so $a x \in J_{2}$. Similarly $x \in J_{2}$ implies that $b x \in J_{1}$. Now put

$$
c=\sum_{r=1}^{n} \frac{n !}{r !(n-r) !} a(b a)^{r-1}(-\lambda)^{n-r} .
$$

By applying the two results just proved one sees that $x \in J_{1}$ implies $c x \in J_{2}$. Since $(b a-\lambda e)^{n}=b c+(-\lambda)^{n} e$, it follows that $b(c x)=-(-\lambda)^{n} x$ for all $x \in J_{1}$. Therefore $L_{b}: J_{2} \rightarrow J_{1}$ is onto. A similar argument based on $(a b-\lambda e)^{n}=$ $c b+(-\lambda)^{n} e$ yields $c(b y)=-(-\lambda)^{n} y$ for all $y \in J_{2}$. Therefore $L_{b}: J_{2} \rightarrow J_{1}$ is one-one.

We have now proved that $L_{b}$ provides an isomorphism of $J_{1}$ with $J_{2}$. In the particular case $n=1$ it proves that $\lambda \neq 0$ is an eigenvalue of $a b$ if and only if it is an eigenvalue of $b a$.

If $\operatorname{dim}(\mathcal{A})<\infty$ and we put $d(a, \lambda, n)=\operatorname{dim}(J(a, \lambda, n))$, then the above lemma establishes that

$$
d(a b, \lambda, n)=d(b a, \lambda, n) \text { for all } \lambda \neq 0, n \geq 1 .
$$


If $\lambda=0$ these identities do not hold even if $\mathcal{A}$ is the algebra of $2 \times 2$ matrices and

$$
a=\left(\begin{array}{cc}
0 & 1 \\
0 & 0
\end{array}\right), \quad b=\left(\begin{array}{ll}
1 & 0 \\
0 & 0
\end{array}\right) .
$$

\section{Transformations of Polynomial Pencils}

Let $S L(2, \mathbf{F})$ denote the multiplicative group of matrices $\left(\begin{array}{ll}a & b \\ c & d\end{array}\right)$ with entries in the field $\mathbf{F}$ and determinant 1. There is an extensive literature on such groups [3], particularly when $\mathbf{F}$ is finite, which we do not assume. It is easy to show that $S L(2, \mathbf{F})$ is generated by $\left(\begin{array}{cc}0 & 1 \\ -1 & 0\end{array}\right)$ and the set of all $\left(\begin{array}{ll}1 & x \\ 0 & 1\end{array}\right)$ where $x \in \mathbf{F}$; see [3, Lemma 6.1.1]. From this it easily follows that the centre of $S L(2, \mathbf{F})$ consists of $\pm\left(\begin{array}{ll}1 & 0 \\ 0 & 1\end{array}\right)$. Therefore the centre contains two elements unless $\mathbf{F}$ has characteristic 2 , in which case it contains only one.

We define $\mathcal{P}_{n}$ to be the space of polynomials with degree at most $n$ in the variable $x \in \mathbf{F}$, where the coefficients lie in the algebra $\mathcal{A}$. In the theory of non-linear eigenvalue problems the spectrum of $p \in \mathcal{P}_{n}$ is defined to be the set

$$
\operatorname{spec}(p)=\{x \in \mathbf{F}: p(x) \text { is not invertible in } \mathcal{A}\} .
$$

We also include a symbolic element $\infty$ in $\operatorname{spec}(p)$ if $p(s)=\sum_{r=0}^{n} a_{r} s^{r}$ and $a_{n}$ is not invertible in $\mathcal{A}$. Clearly $\operatorname{spec}(p)=\operatorname{spec}(b p c)$ whenever $b, c \in \mathcal{A}$ are both invertible.

Lemma 26 Given $g \in G=S L(2, \mathbf{F})$ and $x \in \tilde{\mathbf{F}}:=\mathbf{F} \cup\{\infty\}$, the formula

$$
g \cdot x=\frac{a x+b}{c x+d}
$$

defines a map on $\tilde{\mathbf{F}}$ such that $g .(h . x)=(g h) . x$ for all $g, h \in G$ and all $x \in \tilde{\mathbf{F}}$. Moreover the formula

$$
\left(T_{g} p\right)(x)=(c x+d)^{n} p\left(g^{-1} x\right)
$$

defines a linear map on $\mathcal{P}_{n}$ such that $T_{g} T_{h} p=T_{g h} p$ for all $g, h \in G$ and $p \in \mathcal{P}_{n}$.

Proof This is a direct computation.

If $p(x)=x^{n} a_{n}+\ldots+x a_{1}+a_{0}$ and $g=\left(\begin{array}{cc}0 & 1 \\ -1 & 0\end{array}\right)$ then

$$
\left(T_{g} p\right)(x)=x^{n} a_{0}+\ldots+x a_{n-1}+a_{n}
$$

is often called the reversal of $p$. 
Lemma 27 If $g \in G=S L(2, \mathbf{F}), p \in \mathcal{P}_{n}$ and $q=T_{g}(p)$ then

$$
\operatorname{spec}(q)=g \cdot \operatorname{spec}(p) .
$$

Lemma 28 If $\mathbf{F}$ is infinite, $p \in \mathcal{P}_{n}$ is regular in the sense that $p(x)$ is invertible for some $x \in \mathcal{A}$ and $\operatorname{dim}(\mathcal{A})<\infty$ then there exists $g \in G$ such that $T_{g}(p)=q$ where $q(s)=\sum_{r=0}^{n} a_{r} s^{r}$ and $a_{0}, a_{n}$ are both invertible.

Proof An argument involving determinants proves that the number of $x \in \mathcal{A}$ for which $p(x)$ is not invertible is no larger than $m n$ where $m$ is the dimension of $\mathcal{A}$ and $n$ is the degree of $p$. If $x \neq y$ and $p(x), p(y)$ are both invertible, then there exists $g \in G$ such that $g . x=0$ and $g . y=\infty$. The associated polynomial $q=T_{g}(p)$ then has the required properties.

In many respects it is more natural to consider homogeneous polynomial pencils, i.e. functions

$$
p(x, y)=\sum_{r=0}^{n} x^{r} y^{n-r} a_{r}
$$

where $x, y \in \mathbf{F}$ and $a_{r} \in \mathcal{A}$ for all $r$. The group $S L(2, \mathbf{F})$ acts on such polynomials in a similar fashion, but one no longer needs to consider points at infinity separately. If $\mathbf{F}=\mathbf{R}$ then such a homogeneous polynomial is determined by its values on the unit circle in $\mathbf{R}^{2}$. However, it is possible to analyze the eigenvalue problem in the projective space $P\left(\mathbf{F}^{2}\right)$ directly, without taking a particular parametrization of $P\left(\mathbf{F}^{2}\right)$ by points in $\mathbf{F} \cap\{\infty\}$; see [7].

\section{Factorization of Polynomial Pencils}

Given a monic polynomial pencil with values in $\mathcal{A}$, i.e. an expression of the form

$$
p(x)=\sum_{r=0}^{m} a_{r} x^{r}
$$

where $a_{r} \in \mathcal{A}$ for all $r, a_{m}=e$ and $x \in \mathbf{F}$, there may or may not exist a factorization

$$
p(x)=\prod_{r=1}^{m}\left(x e-b_{r}\right)
$$

such that $b_{r} \in \mathcal{A}$ for all $r$. Putting $m=2$ suppose that $p(x)=x^{2} e-x a+b$ for some $a, b \in \mathcal{A}$ and all $x \in \mathbf{F}$. If $p$ can be factorized as

$$
p(x)=(x e-c)(x e-d)
$$

for some $c, d \in \mathcal{A}$ and all $x \in \mathbf{F}$, a routine calculation shows that

$$
c^{2}-c a+b=0 .
$$


Conversely if this equation is soluble then $p$ can be factorized. See [12, p. 75]. Note also that (6) is equivalent to

$$
d^{2}-a d+b=0
$$

The following example shows that (6) may not be soluble even if $\mathbf{F}=\mathbf{C}$ and $\operatorname{dim}(\mathcal{A})<\infty$.

Example 29 Let $\mathcal{A}$ be the algebra of $n \times n$ complex matrices and let $a \in \mathcal{A}$ be the elementary Jordan matrix such that $a_{r, s}=1$ if $s=r+1$ and $a_{r, s}=0$ otherwise. Then $a^{n-1} \neq 0$ but $a^{n}=0$. Suppose that there exist $c, d \in \mathcal{A}$ such that

$$
x^{2} e-a=(x e-c)(x e-d)
$$

for all $x \in \mathbf{C}$. Then $d=-c$ and $c^{2}=a$. Therefore $c^{2 n-2} \neq 0$ but $c^{2 n}=0$. By writing down the Jordan form of $c$ one readily sees that this is impossible.

If $\operatorname{dim}(\mathcal{A})<\infty$ then (5) and Lemma 22 together imply that

$$
\operatorname{spec}(p)=\operatorname{spec}(c) \cup \operatorname{spec}(d) .
$$

The following example shows that this need not be true if $\mathcal{A}$ is infinite-dimensional.

Example 30 This example requires some familiarity with the spectral theorem for bounded self-adjoint operators. Let $c: \ell^{2}\left(\mathbf{Z}^{+}\right) \rightarrow \ell^{2}\left(\mathbf{Z}^{+}\right)$be the bounded operator given by $(c f)(n)=f(n+1)$ for all $n \geq 0$ and let $d=c^{*}$. Then $c d=e$ but $d c \neq e$. One has

$$
p(x):=x^{2} e-a x+e=(x e-c)(x e-d)
$$

for all $x \in \mathbf{C}$, where $a=c+d$ is self-adjoint with $\operatorname{spec}(a)=[-2,2]$. The spectrum of $p$ is $\{z:|z|=1\}$ but $c, d$ both have spectra equal to $\{z:|z| \leq 1\}$, so

$$
\operatorname{spec}(p) \neq \operatorname{spec}(c) \cup \operatorname{spec}(d) .
$$

However, there exists a different factorization

$$
p(x)=(x e-u)\left(x e-u^{*}\right)
$$

where $u$ is unitary and $u, u^{*}, a$ all commute. For this factorization

$$
\operatorname{spec}(p)=\operatorname{spec}(u)=\operatorname{spec}\left(u^{*}\right)=\{z:|z|=1\} .
$$


Given an algebra $\mathcal{A}$, let $\mathcal{A}_{m}$ denote the algebra of all $m \times m$ matrices with entries in $\mathcal{A}$ and let $e_{m}$ denote the identity element in $\mathcal{A}_{m}$.

Lemma 31 Let $p(x)=\sum_{r=0}^{m} x^{r} a_{r}$ where $x \in \mathbf{F}, a_{r} \in \mathcal{A}$ for all $r$ and $a_{m}=e$. Then $x$ lies in $\operatorname{spec}(p)$ if and only if it lies in the spectrum of the matrix $X \in \mathcal{A}_{m}$ defined by

$$
X_{r, s}= \begin{cases}1 & \text { if } s=r+1 \\ -a_{s-1} & \text { if } r=m \\ 0 & \text { otherwise }\end{cases}
$$

Proof This follows directly from the standard identity

$$
G(x)\left(x e_{m}-X\right)=\left(\begin{array}{cc}
p(x) & 0 \\
0 & e_{m-1}
\end{array}\right) H(x)
$$

in which $G(x), H(x) \in \mathcal{A}_{m}$ are invertible for all $x \in \mathbf{F}$. If $m=4$ they are given by

$$
G(x)=\left(\begin{array}{cccc}
x^{3} e+x^{2} a_{3}+x a_{2}+a_{1} & x^{2} e+x a_{3}+a_{2} & x e+a_{3} & e \\
-e & 0 & 0 & 0 \\
0 & -e & 0 & 0 \\
0 & 0 & -e & 0
\end{array}\right)
$$

and

$$
H(x)=\left(\begin{array}{cccc}
e & 0 & 0 & 0 \\
-x e & e & 0 & 0 \\
0 & -x e & e & 0 \\
0 & 0 & -x e & e
\end{array}\right)
$$

from which the general formula can be inferred.

Our next two results will be used in the proof of Theorem 34.

Lemma 32 (Euclid) Let

$$
p(x)=\sum_{r=0}^{m} a_{r} x^{r}
$$

for all $x \in \mathbf{F}$, where $a_{r} \in \mathcal{A}$ for all $r$ and $a_{m}=e$. Given $d \in \mathcal{A}$ put

$$
q(x)=\sum_{r=0}^{m-1} b_{r} x^{r}
$$

where $b_{r}$ are defined inductively by $b_{m-1}=e$ and

$$
\begin{aligned}
a_{m-1} & =b_{m-2}-b_{m-1} d \\
a_{m-2} & =b_{m-3}-b_{m-2} d \\
\ldots & \\
a_{1} & =b_{0}-b_{1} d .
\end{aligned}
$$


Then

$$
p(x)=q(x)(x e-d)+\text { rem }
$$

for all $x \in \mathbf{F}$ where

$$
\text { rem }=a_{0}+b_{0} d=\sum_{r=0}^{m} a_{r} d^{r}
$$

The proof involves simple substitutions, as does that of the corollary below. We continue with the notation of Lemma 32 .

Corollary 33 Let $\mathcal{A}$ be an algebra of linear operators on some vector space $V$. Let $\mu \in \mathbf{F}$ and $v \in V$. If $d v=\mu v$ and $p(\mu) v=0$ then

$$
p(x) v=q(x)(x e-d) v
$$

for all $x \in \mathbf{F}$.

From this point on we assume that $\mathcal{A}$ is the algebra of all $n \times n$ matrices over a field $\mathbf{F}$ and that $P(x)=\sum_{r=0}^{m} x^{r} A_{r}$ where $A_{m}$ is the identity matrix $I$. The following theorem may be found in [12, Th. 3.21, Cor. 3.22].

Theorem 34 If the polynomial $\operatorname{det}(P(x))$ has $m n$ distinct roots in $\mathbf{F}$ then there exist $C_{1}, \ldots C_{m} \in \mathcal{A}$ such that

$$
P(x)=\left(x I-C_{1}\right)\left(x I-C_{2}\right) \ldots\left(x I-C_{m}\right)
$$

for all $x \in \mathbf{F}$.

Proof By calculating the determinants of every matrix in (7), one sees that the hypothesis is equivalent to the assumption that the $m n \times m n$ matrix $X$ has $m n$ distinct eigenvalues $\lambda_{1}, \ldots, \lambda_{m n}$. The corresponding (right, column) eigenvectors $w_{r} \in \mathbf{F}^{m n}$ of $X$ form a basis in $\mathbf{C}^{m n}$.

If we write the eigenvector $w_{r}$ in the form $\left(u_{1, r}, \ldots, u_{m, r}\right)^{\prime}$, where each $u_{r} \in \mathbf{F}^{n}$, then (7) implies that

$$
\left(\begin{array}{cc}
P\left(\lambda_{r}\right) & 0 \\
0 & e_{m-1}
\end{array}\right) H\left(\lambda_{r}\right) w_{r}=0
$$

SO

$$
P\left(\lambda_{r}\right) u_{1, r}=0 \text { for all } r \in\{1, \ldots, m n\} .
$$

If $u \in \mathbf{F}^{n}$ then using the expansion

$$
\left(\begin{array}{c}
u \\
0 \\
\vdots \\
0
\end{array}\right)=\sum_{r=1}^{m n} \alpha_{r}\left(\begin{array}{c}
u_{1, r} \\
u_{2, r} \\
\vdots \\
u_{m, r}
\end{array}\right)
$$


we deduce that $u=\sum_{r=1}^{m n} \alpha_{r} u_{1, r}$. Therefore $\left\{u_{1, r}\right\}_{r=1}^{m n}$ spans $\mathbf{F}^{n}$. This sequence must contain a basis of $\mathbf{F}^{n}$ and, after relabelling, we may donate it by $\left\{u_{1, r}\right\}_{r=1}^{n}$.

Let $C_{m}$ denote the matrix such that $C_{m} u_{1, r}=\lambda_{r} u_{1, r}$ for all $r$ such that $1 \leq$ $r \leq n$. Corollary 33 implies that

$$
P(x)=Q(x)\left(x I-C_{m}\right) u_{1, r}
$$

for all $1 \leq r \leq n$. Therefore

$$
P(x)=Q(x)\left(x I-C_{m}\right) .
$$

The proof is completed by an induction, noting that $Q(x)$ satisfies the same hypotheses as $P(x)$, but with $m$ replaced by $m-1$.

\section{Quadratic equations in rings}

In this section we study quadratic polynomials in which the variable $s$ or $x$ lies in a (generically non-commutative) ring $\mathcal{R}$ rather than in some base field. If $a, b, c, d \in \mathcal{R}$ the algebraic Riccati equation

$$
s d s+a s+s b+c=0
$$

is of great importance in a variety of contexts, but particularly in optimal control theory; see [24, Chap. 13], where it is assumed that $c, d$ are symmetric real matrices and that $a=b^{\mathrm{T}}$. If $d$ is invertible (which is not generally assumed for the Riccati equation) and we put $x=d s$, then (8) may be rewritten in the form $x^{2}+u x+x v+w=0$ where $u=d a d^{-1}, v=b$ and $w=d c$. We will study this equation within a general ring $\mathcal{R}$.

Theorem 35 Given $u, v, w, u^{\prime}, v^{\prime}, w^{\prime} \in \mathcal{R}$, the identity

$$
x^{2}+u x+x v+w=x^{2}+u^{\prime} x+x v^{\prime}+w^{\prime}
$$

holds for all $x \in \mathcal{R}$ if and only if

$$
w=w^{\prime} \text { and } u-u^{\prime}=v^{\prime}-v \in \mathcal{C} .
$$

Proof The proof of (9) given (10) is a matter of direct substitution. Conversely, given (9), by putting $x=0$ and then $x=e$ one obtains $w=w^{\prime}$ and $u+v=$ $u^{\prime}+v^{\prime}$. It only remains to prove that $c:=u-u^{\prime}\left(=v^{\prime}-v\right)$ lies in $\mathcal{C}$. Given what we have just proved (9) can be reduced to $u x+x v=u^{\prime} x+x v^{\prime}$ and then rewritten in the form $\left(u-u^{\prime}\right) x=x\left(v^{\prime}-v\right)$. This implies that $c x=x c$ for all $x \in \mathcal{A}$, so $c \in \mathcal{C}$. 
Let $p: \mathcal{R} \rightarrow \mathcal{R}$ be a monic quadratic polynomial of the form

$$
p(x)=x^{2}+u x+x v+w .
$$

We define a factorization of $p$ to be an identity of the form

$$
p(x)=(x-a)(x-b),
$$

valid for all $x \in \mathcal{R}$, where $a, b$ is an ordered pair in $\mathcal{R}$. In general a factorization need not exist, and if one does it need not be non-unique.

Example 36 Let

$$
p(x)=x^{2}-\left(\begin{array}{ll}
1 & 0 \\
0 & 0
\end{array}\right) x-x\left(\begin{array}{ll}
0 & 0 \\
0 & 1
\end{array}\right)-\left(\begin{array}{ll}
2 & 0 \\
0 & 2
\end{array}\right)
$$

for all $x \in \mathcal{A}=M(2, \mathbf{C})$. A direct calculation shows that $p(a)=0$ for $a=$ $\left(\begin{array}{ll}2 & 0 \\ 0 & 2\end{array}\right)$. If $p(x)=(x-a)(x-b)$ for some $b \in \mathcal{A}$ and all $x \in \mathcal{A}$ then by putting $x=0$ one sees that $b=-\left(\begin{array}{ll}1 & 0 \\ 0 & 1\end{array}\right)$. By evaluating the other coefficients we deduce that such a factorization does not exist.

Theorem 37 If the centre $\mathcal{C}$ of $\mathcal{R}$ is a field and $p$ has the factorization (11) then that factorization is unique unless $c:=a-b \in \mathcal{C}$, in which case one also has $p(x)=(x-b)(x-a)$ for all $x \in \mathcal{R}$. There is no other factorization.

Proof If $c \in \mathcal{C}$ then it follows immediately that $a$ and $b$ commute and that the second factorization in valid.

Conversely suppose that in addition to (11) one has $p(x)=\left(x-a^{\prime}\right)\left(x-b^{\prime}\right)$ for all $x \in \mathcal{R}$. On expanding both sides of the two factorizations a direct application of Theorem 35 yields

$$
a-a^{\prime}=b^{\prime}-b \in \mathcal{C} \text { and } a b=a^{\prime} b^{\prime} .
$$

If one puts $c=a-a^{\prime}(\in \mathcal{C})$ then these identities imply that $a^{\prime}=a-c, b^{\prime}=b+c$ and $c(a-b)-c^{2}=0$. Assuming that the two factorizations are indeed distinct, $c \neq 0$ and multiplying by $c^{-1}$ yields $a-b=c$. Hence $a^{\prime}=b$ and $b^{\prime}=a$.

\section{The equation $a x-x b=c$}

This section treats a generalization of the Sylvester equation for matrices, conventionally written in the form $A X+X B=C$, and the continuous Lyapunov equation for matrices $A X+X A^{\mathrm{T}}+Q=0$, where the superscript $\mathrm{T}$ denotes 
the matrix transpose. We consider the problem at an algebraic level, but the main novelties are that, motivated by the examples described in Section 10, we do not assume that the algebra is finite-dimensional or that the base field is algebraically closed.

If $a, b, c$ lie in the algebra $M(n, \mathbf{F})$ of $n \times n$ matrices with entries in a field $\mathbf{F}$, the equation $a x-x b=c$ is not always soluble for $x$ within $M(n, \mathbf{F})$. Lemma 46 describes some constraints on the $c$ for which the equation is $a x-x a=c$ is soluble. A detailed analysis of the conditions under which a matrix equation in $M(n, \mathbf{C})$ of the form $a x-x b=c$ is soluble is given in [12, Chap. S2] and [9, 18, 24]. Our own discussion considers the case in which $a, b, c, x$ all lie in an algebra $\mathcal{A}$ over a general field $\mathbf{F}$.

Lemma 38 Given $a, b \in \mathcal{A}$ the following are equivalent.

1. $a, b$ are algebraic and have relatively prime minimum polynomials $p, q$.

2. There exists $f \in \mathcal{P}$ such that $f(a)=e$ and $f(b)=0$.

Proof $1 \Rightarrow 2$. There exist $h, k \in \mathcal{P}$ such that $p h+q k=1$. If we put $f=q k$ then $f(b)=q(b) k(b)=0$ and $f(a)=e-p(a) h(a)=e$.

$2 \Rightarrow 1$. The identity $f(a)=e$ implies that $f \neq 0$ as an element of $\mathcal{P}$, and $f(b)=0$ then implies that $b$ is algebraic with a minimum polynomial $q$; moreover there exists $k \in \mathcal{P}$ such that $f=q k$. Putting $g=1-f$ a similar argument implies that $a$ is algebraic with a minimum polynomial $p$ and that $g=p h$ for some $h \in \mathcal{P}$. Since $p h+q k=1, p, q$ are relatively prime.

We say that $a, b \in \mathcal{A}$ are spectrally disjoint if they are both algebraic and the conditions of Lemma 38 hold.

Theorem 39 If $a, b \in \mathcal{A}$ are spectrally disjoint then $a x-x b=c$ has a unique solution $x \in \mathcal{A}$ for all $c \in \mathcal{A}$.

Proof Let $\mathcal{B}$ be the algebra of all linear maps from $\mathcal{A}$ to $\mathcal{A}$. Define $A, B \in \mathcal{B}$ by $A(x)=a x$ and $B(x)=x b$. If $f \in \mathcal{P}$ is defined as in Lemma 38 then $f(A)(x)=f(a) x=x$ and $f(B)(x)=x f(b)=0$ for all $x \in \mathcal{A}$. Therefore $f(A)=I$ and $f(B)=0$ as elements of $\mathcal{B}$. Since $A, B$ commute we have

$$
I=f(A)-f(B)=(A-B) g(A, B)=g(A, B)(A-B)
$$

where $g$ is a polynomial in two commuting variables; this may be proved by using the particular case

$$
A^{n}-B^{n}=(A-B)\left(A^{n-1}+A^{n-2} B \ldots+A B^{n-2}+B^{n-1}\right) .
$$

Equation (12) implies that $A-B$ is invertible as an element of $\mathcal{B}$, or equivalently one-one and onto as a linear transformation on $\mathcal{A}$. 
Corollary 40 Let $\mathcal{J}$ be an ideal in $\mathcal{A}$. Under the assumptions of Theorem[39 one has $x \in \mathcal{J}$ if and only if $c \in \mathcal{J}$.

Proof The simplest proof involves applying Theorem 39 to the relevant elements of $\mathcal{A} / \mathcal{J}$. The following is a more constructive approach. The statement $(x \in \mathcal{J}) \Rightarrow(c \in \mathcal{J})$ follows directly from the defining properties of an ideal. Conversely (12) implies

$$
x=(A-B)^{-1} c=g(A, B) c .
$$

Since $A$ and $B$ map $\mathcal{J}$ into $\mathcal{J}$, one immediately concludes that $c \in \mathcal{J}$ implies $x \in \mathcal{J}$.

Theorem 39 can be extended to bounded operators on a complex Banach space or even to elements of a Banach algebra; see [17]. The following is a more general version of Theorem 39 .

Theorem 41 If $a, b \in \mathcal{A}$ commute and are spectrally disjoint then $a-b$ is invertible.

Proof Let $f \in \mathcal{P}$ satisfy $f(a)=e$ and $f(b)=0$. There exists a polynomial $g$ in two commuting variables $x, y$ such that

$$
f(x)-f(y)=(x-y) g(x, y) \text { for all } x, y \in \mathbf{F} .
$$

This implies that

$$
e=f(a)-f(b)=(b-a) g(a, b)
$$

so $a-b$ is invertible with inverse $g(a, b)$.

Example 42 Let $V$ denote the vector space of all $m \times n$ matrices with entries in the field $\mathbf{F}$. Given $c \in V, a \in M(m, \mathbf{F})$ and $b \in M(n, \mathbf{F})$, one might wish to solve the equation $a x-x b=c$ for $x \in V$. This problem may be analyzed by using Theorem 41, where $\mathcal{A}$ is taken to be the algebra of all linear maps from $V$ to $V$. If $L, R \in \mathcal{A}$ are defined by $L(v)=a v$ and $R(v)=v b$ then one sees immediately that $L$ and $R$ commute. Moreover $L$ has the same spectrum and minimum polynomial as $a$, while $R$ has the same spectrum and minimum polynomial as $b$. Therefore the equation $a x-x b=c$ is uniquely soluble if $a$ and $b$ are spectrally disjoint.

In order to apply Theorem 39 or 41 in a purely algebraic context we need two lemmas. We define $\mathcal{P}^{*}$ to be the ring of polynomials with entries in the algebraic closure $\mathbf{F}^{*}$ of $\mathbf{F}$. We put $\mathcal{A}^{*}=\mathcal{A} \otimes \mathbf{F}^{*}$ and identify $a \in \mathcal{A}$ with $a \otimes 1 \in \mathcal{A}^{*}$ where 1 is the multiplicative identity in $\mathbf{F}^{*}$. We define $\mathcal{B}$ to be the algebra of all $\mathbf{F}$-linear operators from $\mathcal{A}$ to $\mathcal{A}$ and $\mathcal{B}^{*}$ to be the algebra of all $\mathbf{F}^{*}$-linear operators from $\mathcal{A}^{*}$ to $\mathcal{A}^{*}$. 
Lemma 43 Let $\mathcal{A}$ be an algebra over $\mathbf{F}$ and let $a \neq 0$ be an algebraic element of $\mathcal{A}$. The following four polynomials coincide:

1. the minimum polynomial $p_{1}$ of $a \in \mathcal{A}$;

2. the minimum polynomial $p_{2}$ of $L_{a} \in \mathcal{B}$;

3. the minimum polynomial $p_{3}$ of $a \in \mathcal{A}^{*}$;

4. the minimum polynomial $p_{4}$ of $L_{a} \in \mathcal{B}^{*}$.

Proof The equivalence of items 1 and 2 uses the fact that the homomorphism $a \rightarrow L_{a}$ is one-one. Similarly for items 3 and 4 . We prove the equivalence of items 1 and 3 by using the fact that $\mathcal{P}$ and $\mathcal{P}^{*}$ are principal ideal domains.

Since $p_{1}(a)=0$ in $\mathcal{A}$, this also holds in $\mathcal{A}^{*}$, so $p_{3}$ is a factor of $p_{1}$. Since both are monic, in order to establish that they are equal we need only prove that $\operatorname{deg}\left(p_{3}\right) \geq \operatorname{deg}\left(p_{1}\right)$. Let $P$ be a projection on $\mathbf{F}^{*}$ with range $\mathbf{F}$, where $\mathbf{F}^{*}$ is regarded as a vector space over $\mathbf{F}$. The polynomial $p_{5}=P\left(p_{3}\right) \in \mathcal{P}$ is monic with $p_{5}(a)=0$. Therefore $p_{1}$ is a factor of $p_{5}$ and $\operatorname{deg}\left(p_{3}\right)=\operatorname{deg}\left(p_{5}\right) \geq \operatorname{deg}\left(p_{1}\right)$.

Lemma 44 Let $\mathcal{A}$ be an algebra over a field $\mathbf{F}$ and let $a, b$ be two algebraic elements of $\mathcal{A}$. If $p, q$ are their minimum polynomials then the following are equivalent.

1. $p, q$ are relatively prime in $\mathcal{P}$.

2. $p$ and $q$ are relatively prime in $\mathcal{P}^{*}$.

3. $L_{a}$ and $L_{b}$ have disjoint spectra regarded as operators on $\mathcal{A}^{*}$.

Proof $1 \Rightarrow 2$. The existence of an identity $h p+k q=1$ in $\mathcal{P}$ implies that the same identity holds in $\mathcal{P}^{*}$.

$2 \Rightarrow 1$. Since $p, q$ are relatively prime in $\mathcal{P}^{*}$, there exist $h^{*}, k^{*} \in \mathcal{P}^{*}$ such that $h^{*} p+k^{*} q=1$. Defining the projection $P$ as in the proof of Lemma 43 and putting $h=P\left(h^{*}\right), k=P\left(k^{*}\right)$ we obtain $h p+k q=1$; hence $p, q$ are relatively prime in $\mathcal{P}$.

$2 \Leftrightarrow 3$. It follows from Theorem 4 that both statements are equivalent to $p$ and $q$ having no common zeros.

Example 45 Let $\mathcal{Q}$ denote the algebra of quaternions over a base field $\mathbf{F}$. If

$$
a=a_{0}+a_{1} i+a_{2} j+a_{3} k
$$


then the minimum polynomial of $a$ is

$$
p(z)=z^{2}-2 a_{0} z+\left(a_{0}^{2}+a_{1}^{2}+a_{2}^{2}+a_{3}^{2}\right) .
$$

The field $\mathbf{F}$ is said to be formally real if whenever $a_{r} \in \mathbf{F}$ for $1 \leq r \leq n$ and $\sum_{r=1}^{n} a_{r}^{2}=0$ one has $a_{r}=0$ for all $\leq r \leq n$. Examples are $\mathbf{R}$ and the field of all rational functions in one variable whose coefficients are all real.

If $\mathbf{F}$ is formally real then $\mathcal{Q}$ is a division algebra (skew field). In this case $\operatorname{spec}(a)=\emptyset$ unless $a_{1}=a_{2}=a_{3}=0$, in which case $\operatorname{spec}(a)=a_{0}$. Given $a, b \in \mathcal{Q}$ the equation $a x-x b=c$ has a unique solution $x \in \mathcal{Q}$ for all $c \in \mathcal{A}$ if and only if either $a_{0} \neq b_{0}$ or $a_{1}^{2}+a_{2}^{2}+a_{3}^{2} \neq b_{1}^{2}+b_{2}^{2}+b_{3}^{2}$.

If $a=b$ then Theorem 39 cannot be applied. The next two results provide some insight into this case. A trace on an algebra $\mathcal{A}$ is by definition a nonzero linear functional $\operatorname{tr}: \mathcal{A} \rightarrow \mathbf{F}$ such that $\operatorname{tr}(a b)=\operatorname{tr}(b a)$ for all $a, b \in$ $\mathcal{A}$. Important infinite-dimensional examples arise in the theory of finite von Neumann algebras and free probability theory [21, 22], as well as Example 3.

Lemma 46 Let $\mathcal{A}$ be an algebra with a trace tr. If $a, x, c \in \mathcal{A}$ and $a x-x a=c$ then $c$ satisfies the constraints $\operatorname{tr}\left(a^{m} c\right)=0$ for all $m \geq 0$.

Proof One takes the trace of both sides of the identity

$$
\left[a^{m+1}, x\right]=a^{m}[a, x]+a^{m-1}[a, x] a+\ldots+a[a, x] a^{m-1}+[a, x] a^{m} .
$$

The constraints on $c$ in Lemma 46 are not necessarily independent for different $m$.

Theorem 47 Let $\mathcal{A}=M(n, \mathbf{F})$ and let $d$ be the dimension of the set of $C$ for which $A X-X A=C$ is soluble. Then $0 \leq d \leq n^{2}-n$. The case $d=0$ occurs if $A=I$ while the case $d=n^{2}-n$ occurs if $A$ is the $n \times n$ elementary Jordan matrix $J_{n, \lambda}$ defined by

$$
J_{n, \lambda, r, s}= \begin{cases}\lambda & \text { if } s=r \\ 1 & \text { if } s=r+1 \\ 0 & \text { otherwise }\end{cases}
$$

Proof We define $L: \mathcal{A} \rightarrow \mathcal{A}$ by $L(X)=A X-X A$. Since the rank of a linear transformation does not alter if one increases the base field, we may assume that $\mathbf{F}$ is algebraically closed. We have to prove that

$$
\operatorname{dim}(\operatorname{ker}(L)) \geq n,
$$


with equality in the stated case. We choose a basis of $\mathbf{F}^{n}$ for which $A$ is a block diagonal matrix whose blocks are elementary Jordan matrices. More precisely we assume that the diagonal blocks are $J_{n_{s}, \lambda_{s}}$ where $1 \leq s \leq S$, so that $\sum_{s=1}^{S} n_{s}=n$. Since $\operatorname{ker}(L)$ contains the space of all diagonal block matrices $X$ such that $X A=A X$, we need only prove that the space $V_{s}$ of $n_{s} \times n_{s}$ matrices $X_{s}$ satisfying

$$
X_{s} J_{n_{s}, \lambda_{s}}=J_{n_{s}, \lambda_{s}} X_{s}
$$

has $\operatorname{dim}\left(V_{s}\right)=n_{s}$. A direct calculation shows that $V_{s}$ is the space of $n_{s} \times n_{s}$ upper triangular Toeplitz matrices, which has the required dimension.

\section{Fields of analytic functions}

The ring $\mathcal{P}$ of all polynomials with complex coefficients is an integral domain and its quotient field is the space $\mathcal{Q}$ of all rational functions on $\mathbf{C}$. $\mathcal{Q}$ is not algebraically closed. If $a, b, c \in \mathcal{Q}$ and $a \neq 0$ then the quadratic equation $a x^{2}+b x+c=0$ has a solution in $\mathcal{Q}$ if and only if the discriminant $\Delta=b^{2}-4 a c$ has a square root in $\mathcal{Q}$. This holds if and only if every zero and pole of $f$ has even order.

The elements of $\mathcal{Q}$ have a finite number of poles and so, strictly speaking, are not functions. The proper description involves the theory of germs of analytic functions. To define these we start with expressions of the form

$$
f(z)=z^{m} \sum_{r=0}^{\infty} a_{r} z^{r}
$$

where $m \in \mathbf{Z}$ and $a_{0} \neq 0$ (unless $f$ is identically zero); we assume that the series converges in some neighbourhood $U$ of 0 . We identify two 'germs' $(f, U)$ and $(g, V)$ if $g=f$ on $U \cap V$, and define the sum and product of two germs by pointwise operations, excluding the origin. The set of such germs is a field $\mathbf{F}_{1}$ that contains $\mathcal{Q}$. The series in (13) has a radius of convergence $R \in(0, \infty]$, so the corresponding germ has a canonical representative in which one puts $U=\{z:|z|<R\}$. The ring $\mathbf{E}$ of entire functions on $\mathbf{C}$ is contained in $\mathbf{F}_{1}$ and the factorization theorem of Weierstrass, [14, Section 13.3], implies that its quotient field, also contained in $\mathbf{F}_{1}$, is the field of (germs of) meromorphic functions.

The roots of a polynomial with coefficients in $\mathcal{Q}$ may be described algebraically or geometrically, and the two perspectives are significantly different. Elements of the algebraic closure of $\mathrm{F}$ may be associated with a single-valued branch of a multi-valued analytic function, but geometrically speaking the natural entity is 
the Riemann surface associated with the multi-valued function. The following description presents the elements of the algebraic closure $\mathcal{F}_{2}$ of $\mathcal{Q}$ as analytic functions on a particular domain in $\mathbf{C}$; this domain is far from unique and assigns the origin in $\mathcal{C}$ a status that it does not deserve, but it has the merit of being simple and explicit.

We define $\mathbf{G}$ to consist of all pairs $(f, U)$ where $f$ is an analytic function on a region $U$ of the form

$$
U=\mathbf{C} \backslash \bigcup_{r} S\left(w_{r}\right)
$$

where each $S\left(w_{r}\right)$ is a semi-infinite ray starting at $w_{r}$ and the number of rays is finite or countable; in the second case it is assumed that $\left|w_{r}\right| \rightarrow \infty$ as $r \rightarrow \infty$. If $w=0$ we define $S(w)=[0, \infty)$ and for all other $w$ we define $S(w)=w[1, \infty)$. Each such 'star-shaped' region $U$ is dense in $\mathbf{C}$ and simply connected, and the intersection of any finite number of such $U$ is of the same form.

The sum of $(f, U)$ and $(g, V)$ is defined to be the pointwise sum of $f$ and $g$ with domain $U \cap V$, with a similar definition for the product. We again identify $(f, U)$ and $(g, V)$ as elements of $\mathcal{G}$ if $f=g$ on $U \cap V$. This turns $\mathbf{G}$ into an integral domain, which has the following important property.

Theorem 48 Let

$$
p(z, w)=\sum_{r=0}^{n} a_{r}(w) z^{r}
$$

where $a_{r}$ are entire or meromorphic functions of $w \in \mathbf{C}$ and $a_{n}=1$. Then

$$
p(z, w)=\prod_{r=1}^{n}\left(z-b_{r}(w)\right)
$$

where each $b_{r} \in \mathbf{G}$.

Proof According to [19, Theorem IV.14.2] the roots of the polynomial $z \rightarrow$ $p(z, w)$ are branches of analytic functions of $w$ whose singularities are all algebraic, and the singularities form a discrete set in $\mathbf{C}$. If we denote these by $w_{r}$ then each root has one or more single-valued branches on the set obtained from $\mathbf{C}$ by removing the rays through all $w_{r}$.

Example 49 The solutions of the equation

$$
z^{3}-\left(1-w^{2}\right)=0
$$

are the three functions

$$
f_{r}(w)=\alpha^{r}\left(1-w^{2}\right)^{1 / 3}
$$


where $r=0,1,2$ and $\alpha=\mathrm{e}^{2 \pi i / 3}$. These are different branches of the multivalued cube root. One may define a single-valued branch of $\left(1-w^{2}\right)^{1 / 3}$ on

$$
U=\mathbf{C} \backslash([1,+\infty) \cup(-\infty,-1]) .
$$

by analytically continuing the obvious power series from the unit ball.

Acknowledgements I would like to thank M. Breuning, W. J. Harvey, J. R. Partington, A. Pushnitski and S. Richard for useful comments.

\section{References}

[1] M. F. Atiyah, I. G. Macdonald, Introduction to Commutative Algebra, Addison-Wesley, Reading, Mass., 1969.

[2] N. Bourbaki, Éléments de mathématiques, fasc. 32, Théories spectrales, Chap. 1, 2, Hermann, Paris, 1967.

[3] R. W. Carter, Simple groups of Lie Type, John Wiley and Sons, London, 1989.

[4] G. Dales, Banach Algebras and Automatic Continuity. London Math. Soc. Monographs, Clarendon Press, Oxford, 2000.

[5] E. B. Davies, Linear Operators and their Spectra, Cambridge Univ. Press, 2007.

[6] E. B. Davies, Decomposing the essential spectrum, J. Funct. Anal. 257 (2009) 506-536.

[7] J.-P. Dedier and F Tisseur, Perturbation theory for homogeneous polynomial eigenvalue problems, Linear Algebra Appl., 358 (2003) 71-94.

[8] D. E. Edmunds and W. D. Evans, Spectral Theory and Differential Operators, Oxford Science Publ., Oxford Univ. Press, 1987. Prop. IX.2.5 and Theorem IX.2.4 as a ref for my Theorem 8.

[9] H. Flanders and H. K. Wimmer, On the matrix equations $A X-X B=G$ and $A X-Y B=C$, SIAM J. Appl. Math., 32 (1977) 707-710.

[10] V. Georgescu, A. Iftimovici, $C^{*}$-algebras of quantum Hamiltonians, in Operator Algebras and Mathematical Physics, eds. J.-M. Combes, J. Cuntz, G. A. Elliot, G. Nenciu, H. Siedentop, S. Stratila, Proc. Conf. Operator Algebras and Mathematical Physics, Constanta 2001, Ed. Theta (2003), 123-167. 
[11] V. Georgescu, A. Iftimovici, Localizations at infinity and essential spectrum of quantum Hamiltonians: I. General theory, Rev. Math. Phys. 18 (2006) 417-483.

[12] I. Gohberg, P. Lancaster and L. Rodman, Matrix Polynomials, Acad. Press, New York, 1982.

[13] P. Lancaster, L. Rodman, Algebraic Riccati equations, Oxford Univ. Press, Oxford, 1995.

[14] R. Nevanlinna and V. Paatero, Introduction to Complex Analysis, Addison-Wesley, Reading, Mass., 1964.

[15] V. V. Peller, Hankel Operators and Their Applications, Springer-Verlag, New York, 2003.

[16] A. Pressley and G. Segal, Loop Groups, Oxford Math. Mono., Oxford Univ. Press, 1986.

[17] M. Rosenblum, On the operator equation $B X-X A=Q$, Duke Math. J. 23 (1956) 263269.

[18] W. E. Roth, The equation $A X-Y B=C$ and $A X-X B=C$ in matrices, Proc. Amer. Math. Soc. 3 (1952) 392-396. Amer. Math. Soc., 97 (1952) 392-396.

[19] S. Saks and A. Zygmund, Analytic Functions. Transl E. J. Scott, Monografie Matematyczne, Tom 28, Warsaw, 1952.

[20] B. Sz.-Nagy and C Foiaş, Harmonic Analysis of Operators on Hilbert Space, North-Holland, Amsterdam, 1970.

[21] T. Tao, 254A, Notes 5: Free probability. http://terrytao.wordpress.com/ 2010/02/10/245a-notes-5-free-probability/

[22] D. V. Voiculescu, K. J. Dykema, A. Nica, Free random variables. A noncommutative probability approach to free products with applications to random matrices, operator algebras and harmonic analysis on free groups. CRM Monograph Series, 1. American Mathematical Society, Providence, RI, 1992.

[23] H. Whitney, Complex Analytic Varieties, Addison-Wesley, Reading, Mass., 1972.

[24] K. Zhou, J. C. Doyle and K. Glover, Robust and Optimal Control, Prentice Hall, Upper Saddle River, New Jersey, 1996. 
Department of Mathematics

King's College London

Strand

London, WC2R 2LS

UK

E.Brian.Davies@kcl.ac.uk 